\title{
Efeito de 12 semanas de exercício físico domiciliar na aptidão física de idosas com câncer de mama em hormonioterapia: ensaio clínico randomizado
}

\section{Effect of 12 weeks of home-based physical exercise in the fitness of elderly women with breast cancer: a randomized clinical trial}

(1DBreno Augusto Bormann de Souza Filho' ${ }^{1}$, José Roberto da Silva Júnior ${ }^{2}$, William Serrano Smethurst ${ }^{3}$, Dalmir Cavalcanti dos Santos², Cleber Nascimento do Carmo ${ }^{1}$, (DInês Echenique Mattos ${ }^{1}$, João Guilherme Bezerra Alves ${ }^{2}$

\section{RESUMO}

Objetivo: Avaliar os efeitos de um programa de exercícios físicos domiciliares na aptidão física de idosas com câncer de mama em tratamento. Métodos: Ensaio clínico randomizado com 75 idosas com câncer de mama, realizado em um Hospital Geral do Recife, Brasil, de abril a novembro de 2015. 38 foram alocadas para um programa de exercícios físicos domiciliares, durante 12 semanas, e 37 para o Grupo Controle, o qual recebeu palestras mensais sobre a importância da manutenção de um estilo de vida ativo. Um manual de exercícios físicos e DVD foi previamente desenvolvido e distribuído para o Grupo Intervenção. Foi utilizada a bateria Senior Fitness Test para avaliar a aptidão física. Resultados: Foi observada melhora significativa da força muscular, flexibilidade, equilíbrio e resistência aeróbica no Grupo Intervenção, de acordo com os itens da SFT; Flexão de Antebraço $(13,74$ - 17,10 repetições, $p<0,01)$; Levantar e Sentar $(12,03-14,55$ repetições, $p<0,01)$; Ir e Vir $(6,85-6,00$ segundos, $p<0,01)$, marcha estacionária de 2 minutos (79,08 - 123,37 duplas passadas, $\mathrm{p}<0,01)$; Alcançar as Costas (-8,74 a $-0,42$ centímetros, $p<0,01)$; e Sentar e Alcançar (-6,76 a 4,76 centímetros, $p<0,01)$. Não foram observadas melhoras com relação ao IMC e força de preensão manual. Conclusão: O programa de exercícios físicos domiciliares mostrou eficácia para melhorar a aptidão física em idosas com câncer de mama em tratamento.

Palavras-chave: Neoplasias da Mama, Exercício, Aptidão Física, Idoso

1 Escola Nacional de Saúde Pública - ENSP ${ }^{2}$ Instituto de Medicina Integral Prof. Fernando Figueira - IMIP

${ }^{3}$ Universidade Federal de Pernambuco - UFPE

\section{Correspondência}

Breno Augusto Bormann de Souza Filho E-mail: brenobormann@hotmail.com

Submetido: 08 Julho 2019.

Aceito: 14 Outubro 2019.

\section{Como Citar}

Souza Filho BAB, Silva Júnior JR, Smethurst WS Santos DC, Carmo CN, Mattos IE, et al. Efeito de 12 semanas de exercício físico domiciliar na aptidão física de idosas com câncer de mama em hormonioterapia: ensaio clínico randomizado. Acta Fisiatr. 2019;26(1):6-13

DOI: 10.11606/issn.2317-0190.v26i1a163420

\begin{abstract}
Objective: To evaluate the effects of a home-based physical exercise program on fitness of elderly women with breast cancer. Methods: A randomized clinical trial with 75 elderly patients receiving treatment of breast cancer was developed in a General Hospital of Recife, Brazil, from April to November 2015. 38 were randomized for a home-based physical exercise program during 12 weeks and 37 were Control Group, which received monthly lectures on the importance of maintaining an active lifestyle. An instructional material of physical exercise with a brochure and DVD was previously developed and distributed to the Intervention Group. The Senior Fitness Test was used to evaluated physical fitness. Results: A significant improvement in muscle strength, flexibility, balance and aerobic resistance was observed in the Group Intervention according to the Senior Fitness Test items; 30 -s arm curl (13.74-17.10 repetitions, $\mathrm{p}<0.01)$; 30 -s chair stand $(12.03-14.55$ repetitions, $p<0.01)$; 8 -foot up-and-go $(6.85-6.00$ seconds, $p<0.01)$; 2 -min step test $(79.08-123.37$ past doubles, $p<0.01)$; Back scratch $(-8.74$ to -0.42 centimeters, $p<0.01$ ); Chair sit-and-reach $(-6.76$ to 4.76 centimeters, $p<0.01$ ). However, $\mathrm{BMI}$ and manual grip strength did not show any change in both groups. Conclusion: A homebased physical exercise program showed efficacy to improve physical fitness in elderly patients receiving treatment of breast cancer.
\end{abstract}

Keywords: Breast Neoplasms, Exercise, Physical Fitness, Aged 


\section{INTRODUÇÃO}

O envelhecimento é um importante fator de risco para o desenvolvimento de doenças crônicas, dentre elas o câncer de mama. ${ }^{1}$ Este câncer é a segunda causa de morte entre as mulheres em países desenvolvidos e a principal nos países em desenvolvimento. ${ }^{2,3}$

No Brasil, estima-se aproximadamente 59.700 o número de casos novos para o biênio 2018-2019. ${ }^{4}$ Além disso, o câncer de mama apresenta incidência onze vezes mais elevada entre mulheres com 65 anos ou mais. ${ }^{2}$

Embora os avanços no tratamento oncológico para o câncer de mama aumente a sobrevida do paciente, muitas vezes repercute em efeitos colaterais agudos ou tardios como dores musculares, fadiga, estresse emocional, distúrbios do sono e depressão.5,6

Mudanças no padrão de atividade física e condicionamento físico, sendo esses efeitos evidenciados principalmente na população idosas. ${ }^{7,8}$ Assim, torna-se importante a incorporação de intervenções que promovam o alívio desses efeitos e sintomas durante e após tratamento. ${ }^{9}$

Nesse sentido, a prática regular de atividade física evidencia-se como uma terapêutica importante para a atenuação de efeitos colaterais e melhora da funcionalidade global dos pacientes. ${ }^{10,11}$

Além disso, ser fisicamente ativa diminui o risco de recidivas, aumenta a sobrevida, melhora aspectos sociais e psicológicos como interação social, autoconceito, autoestima, depressão, bem como, aspectos físicos relacionados a aptidão física como flexibilidade, força muscular, agilidade, equilíbrio e resistência aeróbica, o que repercute positivamente na qualidade de vida destas pacientes. ${ }^{12-14}$

Outrossim, a prática de exercícios físicos pode minimizar efeitos negativos de outras morbidades, como obesidade, hipertensão e diabetes, muito frequentes em idosos, que uma vez associada a esses pacientes, aumentam as chances de efeitos negativos na qualidade de vida. ${ }^{15,16}$

Além disso, pacientes fisicamente ativos reduzem o nível de sobrecarga dos familiares/cuidadores atrelados ao cuidado. ${ }^{17}$ Entretanto, embora a realização de atividades físicas pelos pacientes com câncer de mama seja um fator importante para melhora da qualidade de vida e sobrevida, ainda há baixa adesão e aderência dos pacientes durante e após o tratamento, sendo a progressão do câncer e os efeitos colaterais dos tratamentos os principais vilões da não adesão. $^{18}$

Nesse sentido, torna-se necessário o desenvolvimento, incorporação e ampliação, de técnicas e métodos que estimulem e fortaleçam a adesão à prática de atividades físicas nesta população. ${ }^{10,19}$

Dessa forma, o exercício físico domiciliar evidencia-se como uma terapêutica de empoderamento segura, eficaz, reprodutível e de baixo custo para idosos em tratamento para câncer mama. ${ }^{20-24}$

Além disso, a incorporação de programas de exercícios físicos domiciliares apresenta vantagens à saúde pública, especialmente para países em desenvolvimento, como baixo custo, facilidade de aplicação, supervisão, reprodutibilidade, fácil complacência e mitigação das dificuldades de transporte e deslocamento relacionadas ao câncer e tratamentos enfrentados por esta população específica. ${ }^{18,23,24}$

Entretanto, até o presente, não foram identificados estudos realizados no Brasil que abordassem essa temática envolvendo idosas com câncer de mama em tratamento de hormonioterapia.

\section{OBJETIVO}

O presente estudo buscou identificar a eficácia de um programa de exercícios físicos domiciliares na aptidão física de idosas em uso de hormonioterapia para câncer de mama.

\section{MÉTODOS}

Ensaio clínico randomizado, realizado entre abril e novembro de 2015, que avaliou os efeitos de um Programa de exercícios físicos domiciliares realizados com duração de 12 semanas em idosas com câncer de mama em uso de hormonioterapia no Instituto de Medicina Integral Prof. Fernando Figueira (IMIP). O IMIP é o maior hospital do Nordeste do Brasil com 1.082 leitos e seis mil atende pacientes com câncer por ano, incluindo 180 mil procedimentos de câncer (consultas e tratamentos). ${ }^{25}$

O estudo seguiu as recomendações do Consolidated Standards of Reporting Trials (CONSORT), ${ }^{26}$ foi previamente aprovado pelo Comitê de Ética em Pesquisa do IMIP (CAEE: 30356114.1.0000.5201) e registrado no ClinicalTrials.gov (NCT02408133). Todos as participantes foram informadas e assinaram o Termo de Consentimento Livre e Esclarecido.

Os critérios de inclusão foram: idade entre 60 a 74 anos; diagnóstico de câncer de mama em estágio I ou II; em tratamento com terapia hormonal. Os critérios de exclusão foram: contraindicação absoluta para exercícios físicos; ${ }^{27}$ depressão grave de acordo com a Escala de Depressão Geriátrica (GDS-15);28-30 desnutrição (tipos I, II e III), detectada por índice de massa corporal (IMC), de acordo com os critérios da Organização Mundial de Saúde (OMS). ${ }^{31}$

Estimado por meio do programa STATA versão 12. Considerou-se de acordo com estudo realizado com os mesmos objetivos de avaliação da aptidão física em idosos, ${ }^{32}$ um parâmetro de média de 6.0 + 1.0 segundos para o teste "Ir e Vir" no grupo intervenção e 7.0 + 2.0 segundos no grupo controle, indicando níveis aceitáveis de fiabilidade para todos os testes de aptidão funcional. Assim, foi adotado um poder de teste de $80 \%$ e erro tipo I de $10 \%$, totalizando 31 indivíduos em cada grupo. Adicionalmente foi acrescentado $10 \%$ ao total estimado, resultando na necessidade de 35 indivíduos em cada um dos grupos de análise.

Para recrutamento das pacientes, foram seguidas as seguintes etapas: (1) consulta e análise dos prontuários de todas as pacientes com câncer de mama em uso de hormonioterapia acompanhadas no Ambulatório de Oncologia Adulto do IMIP para seleção das pacientes elegíveis com base nos critérios de inclusão; (2) contato telefônico com as idosas que atenderam aos critérios de elegibilidade, sendo informadas sobre a pesquisa e convidadas para reunião presencial e individual, no IMIP, com o objetivo de realizar as avaliações de Depressão Geriátrica, pela GDS-15, e desnutrição, através do IMC, com vistas a atender os critérios de exclusão; (3) As idosas que atendessem a todos os critérios de elegibilidade receberam esclarecimentos sobre a pesquisa e assinaram o Termo de Consentimento Livre e Esclarecido.

A randomização ocorreu a partir de uma alocação computadorizada gerada pelo programa Random Alocation disponível online (http://www.randomized.com). O processo foi realizado por um pesquisador que não participou do recrutamento ou coleta de dados, portanto, sem contato direto com as integrantes da pesquisa. As idosas foram atribuídas, em uma proporção de 1:1, para Grupo Intervenção ou Grupo Controle. O mascaramento do estudo para o braço randomização não foi possível devido à natureza da intervenção.

Para determinar o potencial efeito do exercício físico domiciliar, as pacientes foram randomizadas em dois grupos: Intervenção e Controle.

Grupo Intervenção: foi ofertado ao grupo material instrucional, desenvolvido para o estudo, denominado Ginástica Para Fazer em Casa $^{33}$ (manual impresso com DVD encartado) (disponível como arquivo de suplemento), que contém uma rotina de exercícios semanais progressivos com duração média de 30 minutos a serem realizados autonomamente, por 12 semanas consecutivas, para melhora da amplitude de movimento (19 exercícios), aptidão 
muscular (10 exercícios) e estímulos, além de orientações para a realização de caminhadas.

O manual impresso e a intervenção de treinamento com a demonstração dos exercícios em formato para DVD foram projetados para melhorar a força, flexibilidade, equilíbrio e resistência aeróbica das pacientes. O manual é composto por tópicos como introdução, princípios de segurança e sessões semanais de exercícios progressivos, cada sessão com um conjunto de exercícios para amplitude de movimento, aptidão muscular e aeróbico por meio de caminhadas.

Uma frequência mínima de cinco dias por semana foi sugerida para a execução dessas atividades, respeitando, assim, as recomendações da $\mathrm{OMS}^{34}$ sobre a relação entre atividade/exercício físico e qualidade de vida, bem como o registro, no próprio manual, dos dias da semana e turno em que realizou as atividades propostas.

Todas as orientações e estímulos para adesão foram oferecidos através de uma palestra introdutória e contatos telefônicos realizados 2 vezes por semana (todas as segundas e sextas-feiras) durante as 12 semanas subsequentes ao início do estudo.

Grupo Controle: $\mathrm{O}$ grupo recebeu palestras mensais informativas, por um profissional de saúde (que não integrava o grupo de pesquisa), sobre a importância da manutenção de um estilo de vida ativo e realização de práticas saudáveis.

Em ambos os grupos, não houve interferência por parte dos pesquisadores na rotina de cuidados às pacientes.

Os grupos realizaram avaliações em dois momentos, na linha de base (início da pesquisa) e ao final da 12a semana do estudo (término da pesquisa), sendo elas:

(1) avaliações para caracterização da amostra através de questionários sociodemográficos (idade, raça/cor, estado civil, escolaridade e status ocupacional) e clínico epidemiológicos (tipo histológico do câncer, perfil imunohistoquímico do câncer, estadiamento, se realizou quimioterapia e/ou radioterapia prévia a hormonioterapia e se realizou esvaziamento axilar);

(2) avaliação pré-teste como recomendado para testes físicos submáximos, ${ }^{35}$ onde foi verificada a pressão arterial (PA) (método auscultatório) realizada antes e logo após a aplicação dos testes físicos, com as idosas em repouso por aproximadamente 5 minutos antes da aferição, com duas medidas obtidas num intervalo de 3 minutos e a média calculada em milímetros de mercúrio $(\mathrm{mmHg})$ e igualmente aferida a frequência cardíaca ( $F C$ ) em um minuto antes e depois da avaliação proposta;

(3) avaliação da aptidão física funcional propriamente dita, por meio da bateria de testes Senior Fitness Test; ${ }^{36}$

(4) mensuração da força de preensão manual, através da dinamometria; 37

(5) avaliação do IMC, por meio da antropometria. ${ }^{31}$

O desfecho primário considerado foi a alteração dos valores obtidos na linha de base para aptidão física em relação aos registrados após 12 semanas, avaliada pela variação na performance da escala Senior Fitness Test. A SFT é composta por sete itens de teste, mas neste estudo foram utilizados apenas seis, por haver dois testes que avaliam a mesma valência física, capacidade aeróbica - o teste de andar seis minutos e o teste de marcha estacionária de dois minutos.

Optou-se, portanto, pelo teste de marcha estacionária devido ao espaço físico onde foram realizadas as avaliações ser reduzido.

Nesse sentido, os testes utilizados foram:

(1) Alcançar as Costas, por meio do registro da distância em centímetros entre os dedos médios das mãos posicionadas atrás das costas, para avaliação da flexibilidade do hemicorpo superior;

(2) Sentar e Alcançar, por meio do registro da distância em centímetros do dedo médio da mão ao chão, com objetivo de aferir a flexibilidade do hemicorpo inferior;

(3) Flexão de Antebraço, por meio do registro do número de repetições máximas de flexões realizadas pelo braço dominante em 30 segundos, para avaliação da aptidão muscular superior;
(4) Levantar e Sentar, por meio do registro do número de repetições máximas atingidas em 30 segundos, afim de medir a aptidão muscular inferior;

(5) Ir e Vir em 2,44m, por meio do registro do menor tempo em segundos para contornar um cone posicionado a 2,44 metros da idosa e retornar à posição sentada, para verificar a agilidade e equilíbrio dinâmico;

(6) Marcha Estacionária de 2 min, por meio do registro máximo de duplas passadas estacionárias realizadas em dois minutos, para resistência aeróbia. ${ }^{36}$

Além disso, os testes da SFT apresentam, valores de referência, de acordo com idade e sexo, para classificar os indivíduos avaliados. Cada teste possui valores de referência próprios, que enquadram os indivíduos em percentis de "abaixo da média", "na média" e "acima da média" e, assim, permitem inseri-los em uma possível Zona de Risco para Imobilidade a qual indica vulnerabilidade para perda de mobilidade, quando os valores se apresentem como abaixo do recomendado para a saúde física funcional. ${ }^{36}$

A SFT foi validada com base nos três tipos de evidências propostas pela American Psycological Association: Validade relacionada ao Conteúdo, Critério e Construto. A Validade de Critério encontrado nos diferentes testes em mulheres variou de $r=0.71$ a 0.8138 . A Confiabilidade foi avaliada por meio da Correlação Intraclasse (R), cujos valores variaram de 0.80 a 0.9738 .

Os testes em nosso estudo, foram aplicados em formato de circuito, seguindo a sequência e os protocolos sugeridos pelos autores da SFT. Para maiores detalhes referentes a SFT e seus testes, verificar as referências sugeridas. ${ }^{36,38,39}$

Em nosso estudo, a duração da bateria de testes foi em média de 20 a 25 minutos. Todos os testes foram repetidos nas mesmas condições ao término das 12 semanas do estudo. Como desfechos secundários, foram considerados alterações nas médias quantitativas do Índice de Massa Corporal (IMC) e força de preensão manual. O IMC foi calculado por meio da razão entre o peso da massa magra em quilogramas $(\mathrm{kg})$, pela estatura em metros elevado ao quadrado (m2), utilizando balança eletrônica TANITA $^{\circledR}$ e registrado de acordo com a classificação preconizada pela OMS. ${ }^{31}$

Para o registro da variação no desempenho da força de preensão manual $^{37}$ foi utilizado dinamômetro JAMAR ${ }^{\circledR}$, onde as medidas foram realizadas no braço dominante, com a idosa sentada, ombro aduzido para o lado e o cotovelo fletido 90 - com o antebraço e punho na posição neutra. Os resultados foram registrados em quilogramas e cada medição foi realizada três vezes. Um período de descanso de 1 minuto ocorreu entre cada tentativa. Os valores médios foram usados para análise.

Todas as avaliações foram completamente efetuadas num único momento ao início e término da pesquisa e decorreu nas instalações do hospital onde os idosos foram recrutados e acompanhados.

Para comparação entre os grupos foram calculadas estatísticas descritivas, testes $t$ de Student pareado e não pareado e teste de Mann-Whitney. O teste de Kolmogorov-Smirnov foi utilizado para verificação da normalidade, quando necessário. Testes quiquadrado/Exato de Fisher foram realizados para comparação de frequências. Para análises referentes a adesão e aderência, foi realizado cálculo de frequência. A análise foi realizada com o programa STATA versão 12 e nível de significância de 0.05 .

\section{RESULTADOS}

De acordo com o registro e acompanhamento hospitalar, um total de 114 pacientes recebiam hormonioterapia e foram avaliadas segundo os critérios de elegibilidade. Foram, assim, selecionadas 75 idosas que foram contatadas e convidadas a participar do estudo, onde todas assinaram o Termo de Consentimento Livre e Esclarecido. Dessa forma, após a randomização, 38 idosas foram alocadas para o 
Grupo Intervenção e 37 para o Grupo Controle, seguindo as recomendações para ensaios clínicos do CONSORT. ${ }^{26}$

Não foram observadas diferenças estatísticas entre as idosas dos grupos intervenção e controle em relação às características sociodemográficas e clínicas de base, o que sugere que o processo de randomização entre os grupos apresentou homogeneidade no início da pesquisa (Tabela1).

Tabela 1. Características sociodemográficas da linha de base da população do estudo

\begin{tabular}{|c|c|c|c|}
\hline & $\begin{array}{c}\text { Grupo } \\
\text { Intervenção } \\
\text { (n= 38) N } \\
(51 \%)\end{array}$ & $\begin{array}{c}\text { Grupo } \\
\text { Controle } \\
(\mathrm{n}=37) \mathrm{N} \\
(49 \%)\end{array}$ & P-valor \\
\hline Idade, anos: media (DP) a & $66.8(3.5)$ & $66.6(3.8)$ & 0,920 \\
\hline \multicolumn{4}{|l|}{ Raça/Cor ${ }^{c}$} \\
\hline Branca & $14(36.8)$ & $23(62.2)$ & 0,076 \\
\hline Negra/Preta & $5(13.2)$ & $5(13.5)$ & \\
\hline Parda & $18(47.4)$ & $8(21.6)$ & \\
\hline Outro & $1(2.6)$ & $1(2.7)$ & \\
\hline \multicolumn{4}{|l|}{ Estado Civil ${ }^{c}$} \\
\hline Solteira & $10(26.3)$ & $10(27.0)$ & 0,560 \\
\hline Casada & $16(42.1)$ & $14(37.8)$ & \\
\hline Divorciada & $7(18.4)$ & $4(10.8)$ & \\
\hline Viúva & $5(13.2)$ & $9(24.3)$ & \\
\hline \multicolumn{4}{|l|}{ Escolaridade $^{\mathrm{b}}$} \\
\hline Até Fundamental & $8(21.1)$ & $17(45.9)$ & 0,088 \\
\hline Fundamental Completo & $5(13.2)$ & $6(16.2)$ & \\
\hline Ensino Médio & $11(28.9)$ & $7(18.9)$ & \\
\hline Ensino Superior & $14(36.8)$ & $7(18.9)$ & \\
\hline \multicolumn{4}{|l|}{ Status Ocupacional c } \\
\hline Empregada & $8(21.1)$ & $8(21.6)$ & 0,927 \\
\hline Desempregada & $2(5.3)$ & $3(8.1)$ & \\
\hline Aposentada & $28(73.7)$ & $26(70.3)$ & \\
\hline Fumante $(\operatorname{sim})^{c}$ & $3(7.9)$ & $5(13.5)$ & 0,480 \\
\hline Consumo de álcool (sim) ${ }^{\text {b }}$ & $9(23.7)$ & $7(18.9)$ & 0,615 \\
\hline Comorbidade $(\operatorname{sim})^{b}$ & $31(81.6)$ & $30(81.1)$ & 0,956 \\
\hline \multicolumn{4}{|l|}{ Tipo de morbidade ${ }^{c}$} \\
\hline Diabetes Tipo 2 & $1(3.2)$ & $1(3.3)$ & 0,506 \\
\hline Hipertensão & $17(54.8)$ & $11(36.7)$ & \\
\hline Osteoartrite & $1(3.2)$ & $1(3.3)$ & \\
\hline$>2$ morbidades & $12(31.6)$ & $17(45.9)$ & \\
\hline \multicolumn{4}{|l|}{$\begin{array}{l}\text { Tipo Histológico do } \\
\text { Câncer de Mama }{ }^{c}\end{array}$} \\
\hline Carcinoma Ductal Invasivo & $33(86.8)$ & $35(94.6)$ & 0,592 \\
\hline Carcinoma Lobular & $3(7.9)$ & $2(5.4)$ & \\
\hline Carcinoma Mucinoso & $2(5.2)$ & - & \\
\hline \multicolumn{4}{|l|}{ Estagio do Câncer ${ }^{b}$} \\
\hline I & $15(39.5)$ & $18(48.6)$ & 0,424 \\
\hline II & $23(60.5)$ & $19(51.4)$ & \\
\hline \multicolumn{4}{|l|}{ Tratamento Hormonalc } \\
\hline Tamoxifeno & $21(55.3)$ & $24(64.9)$ & 0,646 \\
\hline Anastrozol & $10(26.3)$ & $9(24.3)$ & \\
\hline Exemestano & 7 (18.4) & $4(10.8)$ & \\
\hline $\begin{array}{l}\text { Quimioterapia Previa a } \\
\text { Terapia Hormonal }^{b}\end{array}$ & $20(52.6)$ & $20(54.1)$ & 0,902 \\
\hline $\begin{array}{l}\text { Radioterapia previa a } \\
\text { Terapia Hormonal }^{\text {b }}\end{array}$ & $25(65.8)$ & $19(51.4)$ & 0,204 \\
\hline \multicolumn{4}{|l|}{ Tipo de Cirurgia Realizada } \\
\hline Quadrantectomia & $23(60.5)$ & $19(51.4)$ & 0,424 \\
\hline Mastectomia & $15(39.5)$ & $18(48.6)$ & \\
\hline Esvaziamento Axilar ${ }^{\mathrm{b}}$ & $21(55.3)$ & $23(62.2)$ & 0,544 \\
\hline
\end{tabular}

${ }^{a}$ Student'T test; ${ }^{b} \times 2$ test; $^{c}{ }^{\text {Fisher's exact test }}$
A Tabela 2 apresenta valores médios de IMC, força de preensão manual e da bateria SFT no pré e pós-teste, intra e intergrupos. Para o IMC e força de preensão manual não foram encontradas diferenças significativas intra e intergrupos. Entretanto, para a SFT, observou-se melhora nas variáveis de aptidão física para todos os testes no Grupo Intervenção $(p=0.01)$ e um declínio $(p=0.01)$ no Grupo Controle (Tabela 2).

Tabela 2. Resultados intra e intergrupos dos indivíduos da amostra

\begin{tabular}{lcccc}
\hline \multicolumn{1}{c}{ Avaliações } & Grupos & $\begin{array}{c}\text { Linha de } \\
\text { Base media } \\
\text { (DP) }\end{array}$ & $\begin{array}{c}\text { Após 12 } \\
\text { Semanas } \\
\text { media (DP) }\end{array}$ & $\begin{array}{c}\boldsymbol{P} \text { - } \\
\text { valor }\end{array}$ \\
\hline Forca de & Intervenção & $19.79(4.18)$ & 21.47 & 0,093 \\
Preensão, kg a & & $(3.88)$ & \\
& Controle & $21.78(4.08)$ & 20.16 & 0,072 \\
& P-valor & 0,103 & 0,091 & \\
IMC $^{\text {b }}$ & Intervenção & $29.32(3.92)$ & 28.81 & $0.280^{c}$ \\
& Controle & $30.08(4.67)$ & 30.30 & 0,580 \\
& P-valor & 0,230 & 0,070 & \\
& \multicolumn{4}{c}{ Testes da SFT }
\end{tabular}

\begin{tabular}{|c|c|c|c|c|}
\hline $\begin{array}{l}\text { Flexibilidade } \\
\text { superior }^{b}\end{array}$ & Intervenção & $-8.74(10.63)$ & $-0.42(8.18)$ & $<0.01$ \\
\hline $\begin{array}{l}\text { (Alcançar as } \\
\text { Costas - cm) }\end{array}$ & Controle & $-8.84(9.89)$ & $-14.57(9.80)$ & $<0.01$ \\
\hline & $P$-valor & 0,480 & $<0.01$ & \\
\hline $\begin{array}{l}\text { Flexibilidade } \\
\text { inferior }{ }^{\text {a }}\end{array}$ & Intervenção & $-6.76(8.84)$ & $4.76(8.12)$ & $<0.01$ \\
\hline $\begin{array}{l}\text { (Sentar e } \\
\text { Alcançar - cm) }\end{array}$ & Controle & $-3.12(7.34)$ & $-11.85(8.47)$ & $<0.01$ \\
\hline & $P$-valor & 0,102 & $<0.01$ & \\
\hline $\begin{array}{l}\text { Forca } \\
\text { Superior }{ }^{b}\end{array}$ & Intervenção & 13.74 (3.37) & $17.1(4.09)$ & $<0.01$ \\
\hline $\begin{array}{l}\text { (Flexão de } \\
\text { Antebraço - 30') }\end{array}$ & Controle & $14.51(4.21)$ & $12.49(3.33)$ & 0,012 \\
\hline & $P$-valor & 0,810 & $<0.01$ & \\
\hline $\begin{array}{l}\text { Forca } \\
\text { Inferior b }\end{array}$ & Intervenção & $12.03(2.84)$ & $14.55(3.12)$ & $<0.01$ \\
\hline $\begin{array}{l}\text { (Levantar e } \\
\text { Sentar - 30') }\end{array}$ & Controle & $12.81(2.88)$ & 11.16 (2.66) & $<0.01$ \\
\hline & P-valor & 0,880 & $<0.01$ & \\
\hline $\begin{array}{l}\text { Agilidade/ } \\
\text { Equilíbrio }^{\text {b }}\end{array}$ & Intervenção & $6.85(1.28)$ & $6.00(0.96)$ & $<0.01$ \\
\hline (Ir e Vir - min.) & Controle & $6.35(0.88)$ & $7.16(0.92)$ & $<0.01$ \\
\hline & $P$-valor & 0,970 & $<0.01$ & \\
\hline $\begin{array}{l}\text { Resistencia } \\
\text { Aeróbia }{ }^{b} \\
\text { (Marcha }\end{array}$ & Intervenção & 79.08 (20.63) & $123.37(23.32)$ & $<0.01$ \\
\hline $\begin{array}{l}\text { Estacionaria de } \\
2 \mathrm{~min} \text {.) }\end{array}$ & Controle & 83.94 (21.68) & 74.35 (17) & 0,019 \\
\hline & $P$-valor & 0,840 & $<0.01$ & \\
\hline
\end{tabular}

${ }^{a}$ Mann-Whitney test; ${ }^{b}$ Student'T paired test; ${ }^{c}$ Student'T not paired test

Na Tabela 3 observa-se a distribuição percentual das idosas segundo idade e de acordo com os valores de referência da SFT para a classificação "abaixo da média", "na média" ou "acima da média" de cada variável de teste.

No momento inicial não foram encontradas diferenças intergrupos para nenhuma variável avaliada pela SFT. Entretanto, quando comparados os grupos após 12 semanas, foram observadas diferenças para todos os testes, com ganhos de aptidão para o Grupo Intervenção $(p<0.001)$. Além disso, o Grupo Controle evidenciou um aumento de idosas classificadas com menor aptidão física. 
Tabela 3. Distribuição dos indivíduos da amostra de acordo com a referenda do padrão SFT

\begin{tabular}{|c|c|c|c|c|c|c|}
\hline \multirow[b]{2}{*}{ Variável } & \multicolumn{3}{|c|}{ Linha de Base Grupo } & \multicolumn{3}{|c|}{ Após 12 Semanas Grupo } \\
\hline & $\begin{array}{c}\text { Grupo } \\
\text { Controle } \\
\text { (n= 37) N(\%) }\end{array}$ & $\begin{array}{c}\text { Grupo } \\
\text { Intervenção } \\
(n=38) \quad N(\%)\end{array}$ & P-valor & $\begin{array}{c}\text { Grupo } \\
\text { Controle } \\
(n=37) N(\%)\end{array}$ & $\begin{array}{c}\text { Grupo } \\
\text { Intervenção } \\
(n=38) N(\%)\end{array}$ & P-valor \\
\hline \multicolumn{7}{|l|}{ Flexibilidade Superior } \\
\hline \multicolumn{7}{|l|}{ (Alcançar as Costas) } \\
\hline Acima da media & $9(24.3)$ & $9(23.7)$ & $0.891^{b}$ & $2(5.4)$ & $15(39.5)$ & $<0.001^{b}$ \\
\hline Na media & $4(10.8)$ & $6(15.8)$ & & $3(8.1)$ & $8(21.1)$ & \\
\hline Abaixo da media & $24(64.9)$ & $23(60.5)$ & & $32(86.5)$ & $15(47.4)$ & \\
\hline \multicolumn{7}{|l|}{ Flexibilidade Inferior } \\
\hline \multicolumn{7}{|l|}{ (Sentar e Alcançar) } \\
\hline Acima da media & $5(13.5)$ & $2(5.3)$ & $0.232^{b}$ & $1(2.7)$ & $12(31.6)$ & $<0.001^{b}$ \\
\hline Na media & $25(67.6)$ & $23(60.5)$ & & $19(51.3)$ & $23(60.5)$ & \\
\hline Abaixo da media & 7 (18.9) & $13(24.2)$ & & $17(45.9)$ & $3(7.9)$ & \\
\hline \multicolumn{7}{|l|}{ Força Superior } \\
\hline \multicolumn{7}{|l|}{ (Flexão de Antebraço) } \\
\hline Acima da media & $6(16.2)$ & $2(5.3)$ & $0.363^{b}$ & $2(5.4)$ & $15(39.5)$ & $<0.001^{b}$ \\
\hline Na media & $23(62.2)$ & $26(68.4)$ & & $13(35.1)$ & $18(47.4)$ & \\
\hline Abaixo da media & $8(21.6)$ & $10(26.3)$ & & $22(59.5)$ & $5(13.1)$ & \\
\hline \multicolumn{7}{|l|}{ Força Inferior } \\
\hline \multicolumn{7}{|l|}{ (Levantar e Sentar) } \\
\hline Acima da media & $6(16.2)$ & $3(7.9)$ & $0.445^{b}$ & $1(2.7)$ & $22(57.9)$ & $<0.001^{b}$ \\
\hline Na media & $11(29.7)$ & $10(26.3)$ & & $4(10.8)$ & $6(15.8)$ & \\
\hline Abaixo da media & $20(54)$ & $25(65.8)$ & & $32(86.5)$ & $10(26.3)$ & \\
\hline \multicolumn{7}{|l|}{ Agilidade/Equilíbrio } \\
\hline \multicolumn{7}{|l|}{ (Ir e Vir) } \\
\hline Acima da media & $0(0)$ & $0(0)$ & $0.168^{b}$ & $0(0)$ & $2(5.3)$ & $<0.001^{b}$ \\
\hline Na media & $22(59.4)$ & $16(42.1)$ & & $9(24.3)$ & $25(65.8)$ & \\
\hline Abaixo da media & $15(40.5)$ & $22(57.9)$ & & $28(75.7)$ & $11(28.9)$ & \\
\hline \multirow{2}{*}{\multicolumn{7}{|c|}{$\begin{array}{l}\text { Resistencia Aeróbia } \\
\text { (Marcha Estacionaria) }\end{array}$}} \\
\hline & & & & & & \\
\hline Acima da media & 7 (18.9) & $5(13.1)$ & $0.618^{a}$ & $1(2.7)$ & $28(73.7)$ & $<0.001^{b}$ \\
\hline Na media & $19(51.3)$ & $18(47.4)$ & & $19(51.3)$ & $10(26.3)$ & \\
\hline Abaixo da media & $11(29.7)$ & $15(39.5)$ & & 17 (45.9) & $0(0)$ & \\
\hline
\end{tabular}

${ }^{a} \times 2$ test; ${ }^{b}$ Fisher's exact test

Nesse sentido, o programa de exercícios físicos melhorou a aptidão física do Grupo Intervenção, o que resultou no aumento percentual de idosas fora da zona de risco para imobilidade para todos os testes (10.5\% para $60.5 \%$ ). Para o grupo controle, foi observada diminuição da aptidão física em todas as variáveis, o que aumentou o percentual de idosas (91.9\% para $100 \%$ ) em uma ou mais variável classificada na zona de risco para imobilidade funcional após as 12 semanas (Tabela 4).

Tabela 4. Distribuição dos indivíduos do estudo de acordo com o Risco de Imobilidade Funcional

\begin{tabular}{|c|c|c|c|c|}
\hline & \multicolumn{2}{|c|}{ Linha de Base } & \multicolumn{2}{|c|}{ Após 12 semanas } \\
\hline & $\begin{array}{c}\text { Grupo } \\
\text { Controle } \\
\text { (n=37) } \\
\text { N (\%) }\end{array}$ & $\begin{array}{c}\text { Grupo } \\
\text { Intervenção } \\
(n=38) \\
N(\%)\end{array}$ & $\begin{array}{c}\text { Grupo } \\
\text { Controle } \\
\text { (n= 37) } \\
\text { N (\%) }\end{array}$ & $\begin{array}{c}\text { Grupo } \\
\text { Intervenção } \\
\text { (n= 38) } \\
\text { N (\%) }\end{array}$ \\
\hline Em 5 zonas de risco & $0(0)$ & $0(0)$ & $1(2.7)$ & $0(0)$ \\
\hline Em 4 zonas de risco & $1(2.7)$ & $3(7.9)$ & $5(13.5)$ & $0(0)$ \\
\hline Em 3 zonas de risco & $3(8.1)$ & $7(18.4)$ & $6(16.2)$ & $0(0)$ \\
\hline Em 2 zonas de risco & $14(37.8)$ & $14(36.8)$ & $20(54.1)$ & $7(18.4)$ \\
\hline Em 1 zona de risco & $16(43.2)$ & $10(26.3)$ & $5(13.5)$ & $8(21.1)$ \\
\hline Fora da zona de risco & 3 & $4(10.5)$ & $0(0)$ & $23(60.5)$ \\
\hline
\end{tabular}

Potenciais efeitos adversos da intervenção não foram relatados pelas participantes. Os eventos relatados espontaneamente foram dores musculares, que cessaram em média com uma semana e meia de intervenção para o Grupo Intervenção. Este achado pode ser justificado pelo estilo de vida sedentário das participantes antes, durante e após tratamento para câncer de mama. ${ }^{10,14}$

Além disso, foi relatado pelas idosas do Grupo Intervenção que a única prática de exercícios físicos realizada pelas mesmas, durante o período do estudo, foi aquela indicada no manual. Com relação às idosas do Grupo Controle, foi relatado que não adotaram a prática regular de atividades físicas durante o mesmo período.

A assiduidade média das idosas do Grupo Intervenção analisada de acordo com a realização completa dos exercícios recomendados nas sessões do programa de treinamento (amplitude de movimento; aptidão muscular; e caminhadas) com frequência mínima de cinco dias da semana, foi de $81,5 \%(n=31)$. Além disso, foi observado um declínio com relação a aderência das idosas a partir da sétima semana do Programa (dados apenas informativos, não disponíveis nas tabelas).

Esses dados sugerem que pode ser interessante, além dos contatos telefônicos, a realização de uma avaliação física presencial na sexta semana de realização do Programa, com objetivo motivacional. 


\section{DISCUSSÃO}

Este estudo randomizado controlado investigou de forma pioneira no Brasil os efeitos de um programa de exercícios físicos domiciliares sobre a aptidão física de idosas em tratamento hormonioterápico para câncer de mama, realizado através de um manual instrucional de forma autônoma. Foi observado que esta foi uma estratégia eficaz no ganho da aptidão física, com melhora significativa para todos os itens avaliados através da bateria de testes SFT. Com o aumento da sobrevida das pacientes com câncer de mama, a capacidade de desfrutar um estilo de vida ativo e independente dependerá em grande parte da manutenção do nível de aptidão física. ${ }^{14}$

Nas idosas com câncer de mama, uma das principais preocupações consiste na manutenção da mobilidade e da autonomia, necessárias para realizar de forma independente as atividades diárias. ${ }^{40,41}$ Nesse sentido, estudos têm investigado cada vez mais o efeito do exercício sobre a aptidão física em pacientes com câncer de mama. ${ }^{14,42}$

Dentre os atributos relacionados à aptidão física, a flexibilidade é um elemento que pode sofrer alterações referentes ao processo de envelhecimento ${ }^{36}$ e ao tratamento oncológico. ${ }^{10}$ Pacientes com câncer de mama podem ter a flexibilidade ainda mais reduzida, devido aos processos cirúrgicos comumente associados ao tratamento. ${ }^{9}$ A perda da flexibilidade, de modo geral para os hemicorpos superior e inferior, contribui para a dependência física, uma vez que o indivíduo poderá apresentar limitações para a realização de atividades da vida diária como subir degraus, curvar-se, abaixar-se, levantar objetos, pentear os cabelos e vestir-se. ${ }^{43}$

Neste estudo, observamos diferenças com relação à flexibilidade do hemicorpo superior e inferior por meio dos testes "Alcançar as Costas" e "Sentar e Alcançar" respectivamente. O Grupo Intervenção apresentou melhora e resultados acima dos valores de referência, enquanto o Grupo Controle apresentou valores abaixo dos parâmetros previstos para idade e sexo.

Esses achados são promissores uma vez que evidencia a eficácia de 12 semanas do Programa de exercícios físicos domiciliares em contraponto a outros métodos de treino até então indicados para a flexibilidade como o pilates ${ }^{44}$ e yoga. ${ }^{45}$ Bertoli $^{44}$ verificou o efeito de 12 semanas de treinamento de pilates de solo nas variáveis funcionais de idosas saudáveis, entre elas a flexibilidade, e observou que após a avaliação com o teste "Alcançar as Costas" não obteve melhoras significativas.

Em consonância, Loudon et al. ${ }^{45}$ avaliaram o efeito de 8 semanas de yoga com sessões diárias via DVD e encontros presenciais para a amplitude de movimento do ombro em 23 mulheres com câncer de mama e não foram observadas melhoras significativas.

É provável que a forma como foi trabalhada a flexibilidade em nosso estudo - de forma progressiva para os exercícios de amplitude de movimento associados aos de aptidão muscular - justifiquem esse efeito positivo, o que reforça a hipótese de que a aptidão física deve ser trabalhada através de atividades corporais globais e combinadas para essa população. ${ }^{46}$

Outro componente importante da aptidão física é a força muscular. Seu declínio, associado ao processo de envelhecimento, estilo de vida sedentário e uso de tratamentos antineoplásicos, pode produzir efeitos indesejáveis na capacidade de realizar atividades do dia-a-dia. ${ }^{46}$ Assim, preservar a força muscular e realizar sua mensuração, tanto para membros superiores quanto inferiores, torna-se essencial na avaliação da aptidão física e elaboração de programas de atividades físicas para idosas com câncer de mama. ${ }^{36}$

Dentre a variedade de métodos de prescrição de exercícios utilizados para ganho de força muscular, o treino resistido supervisionado ainda é tradicionalmente o mais adotado. ${ }^{10}$ Entretanto, em nosso estudo, a partir da adoção de método domiciliar não supervisionado, identificamos por meio do teste
"Flexão de Antebraço" e "Levantar e Sentar", ganhos significativos de força relacionado aos hemicorpos superior e inferior para o Grupo Intervenção e piora do Grupo Controle após 12 semanas.

Em investigação, 61 idosas sem diagnóstico de câncer foram divididas em dois grupos: o primeiro composto por mulheres fisicamente ativas há mais de seis meses e o outro por iniciantes, ambos integrantes de um programa de exercícios físicos comunitário supervisionado realizado duas vezes por semana. Verificou-se um efeito satisfatório sobre a variável força para membros superiores, porém, o mesmo não foi observado com relação a força de membros inferiores. ${ }^{47}$

Embora haja grande recomendação da Organização Mundial de Saúde para ampliação de espaços para realização de atividades físicas ao ar livre, ${ }^{19}$ idosas com câncer de mama e sob efeito da progressão da doença e/ou colaterais do tratamento, tendem a apresentar barreiras motivacionais que as impedem de integrarem programas de exercícios físicos comunitários. ${ }^{18}$

Nesse sentido, destacamos que a realização de exercícios físicos domiciliares em apenas 12 semanas, conforme sugerido no nosso estudo, evidenciou que esse método adotado em ambiente domiciliar, com auxílio de estímulos a distância via telefone, pode ser uma alternativa eficaz e de baixo custo a ser adotada pelas equipes de saúde no que tange a melhora da força de membros superiores e inferiores de idosas com câncer de mama no combate ao declínio funcional.

As reduções da massa e força muscular associadas ao déficit de equilíbrio aumentam o risco de quedas nos idosos. ${ }^{48}$ Dessa forma, a variável equilíbrio torna-se um aspecto importante a ser avaliado nesse tipo de população com câncer. Em nosso estudo, verificamos um efeito positivo nessa variável para o Grupo Intervenção e um declínio para o Grupo Controle. Além disso, no Grupo Intervenção, $65.8 \%$ das idosas obtiveram valores correspondentes a percentis "na média", quando considerados os critérios normativos do teste "Ir e Vir"; enquanto no Grupo Controle $57.9 \%$ das idosas foram classificadas como "abaixo da média", corroborando achados de outro estudo. ${ }^{47}$

Referente à resistência aeróbia avaliada por meio do teste "Marcha Estacionária de 2 minutos", observou-se diferença entre os grupos com ganhos para o Grupo Intervenção. De acordo com valores normativos de referência padrão, $73.7 \%$ das idosas do Grupo Intervenção encontravam-se "acima da média", em contraponto a 51.3\% das idosas do Grupo Controle, classificadas "na média".

Em estudo que analisou o efeito de um programa de exercícios sistematizado de 12 versus 24 semanas, não foram verificados ganhos significativos na capacidade aeróbia ao comparar os indivíduos no início do programa e ao final das 12 semanas. ${ }^{49} \mathrm{~A}$ melhora da resistência aeróbia em nossos achados pode ser atribuída ao efeito cumulativo do programa por meio das sessões de exercícios funcionais para amplitude de movimento e resistência muscular do manual instrucional, associado a estímulos para caminhadas voluntárias. ${ }^{46}$

Embora não tenhamos observado alterações estatisticamente significativas para o IMC e força de preensão manual através do Programa de Exercícios Físicos Domiciliares, para os demais componentes da aptidão física avaliados, por meio da SFT, verificaram-se ganhos para o Grupo Intervenção. Dessa forma, nosso estudo fortalece a hipótese da utilização deste método como intervenção inicial ao processo de reabilitação das idosas com câncer de mama em hormonioterapia, otimizando resultados bem como empoderando essa população para o autocuidado apoiado.

Além disso, até onde vai nosso conhecimento, este também foi o primeiro estudo a utilizar a SFT como instrumento de mensuração da capacidade física funcional em idosas com câncer de mama em uso de homonioterapia. No entanto, existem potenciais limitações neste estudo como: baixo recrutamento de pacientes devido aos rigorosos critérios de inclusão e exclusão; não utilização de marcadores 
bioquímicos e hemodinâmicos que poderiam ser fatores de impedimento ou não da prática de atividades físicas; a relação temporal de 12 semanas de prática de exercícios não permite avaliar os efeitos em longo prazo desse tipo de intervenção sobre uma resposta sistêmica; a falta de um grupo controle negativo (indivíduos saudáveis) para avaliar se o resultado encontrado poderia ter sido o efeito do programa de exercícios na condição clínica do paciente.

Dessa forma, é importante ressaltar que nossos resultados não podem ser generalizáveis para população saudável, de outras faixas etárias ou com outras doenças crônicas, assim como para outros tipos de câncer e tratamentos.

\section{CONCLUSÃO}

Nosso estudo demonstra a eficácia de um Programa de 12 semanas de exercícios físicos domiciliares não supervisionado para idosas com câncer de mama, realizado de forma autônoma e independente.

Nesse sentido, nossos achados são encorajadores, pois indicam que programas de exercícios físicos domiciliares podem ser realizados de maneira eficaz e segura por meio de materiais instrucionais e estímulos telefônicos semanais. Isto tem implicações importantes para a futura disseminação deste tipo de intervenção, com vistas na promoção da saúde bem como para pesquisas futuras.

\section{REFERÊNCIAS}

1. American Cancer Society. Cancer Facts \& Figures 2018. Atlanta: American Cancer Society; 2018.

2. Bray F, Ferlay J, Soerjomataram I, Siegel RL, Torre LA, Jemal A. Global cancer statistics 2018: GLOBOCAN estimates of incidence and mortality worldwide for 36 cancers in 185 countries. CA Cancer J Clin. 2018;68(6):394-424. DOI: https://doi.org/10.3322/caac.21492

3. Siegel RL, Miller KD, Jemal A. Cancer statistics, 2018. CA Cancer J Clin. 2018;68(1):7-30. DOI: https://doi.org/10.3322/caac.21442

4. Instituto Nacional de Câncer. Estimativa 2018: incidência de câncer no Brasil. Rio de Janeiro: INCA; 2017.

5. Canário AC, Cabral PU, Paiva LC, Florencio GL, Spyrides MH, Gonçalves AK. Physical activity, fatigue and quality of life in breast cancer patients. Rev Assoc Med Bras (1992). 2016;62(1):38-44. DOI: https://doi.org/10.1590/1806-9282.62.01.38

6. Bener A, Alsulaiman R, Doodson L, Agathangelou T. Depression, hopelessness and social support among breast cancer patients: in highly endogamous population. Asian Pac J Cancer Prev. 2017;18(7):1889-96.

https://doi.org/10.22034/APJCP.2017.18.7.1889

7. Ahmed AE, Alharbi AG, Alsadhan MA, Almuzaini AS, Almuzaini HS, Ali $Y Z$, et al. The predictors of poor quality of life in a sample of Saudi women with breast cancer. Breast Cancer (Dove Med Press). 2017;9:51-8. DOI: https://doi.org/10.2147/BCTT.S125206

8. Tiezzi MF, Andrade JM, Romão AP, Tiezzi DG, Lerri MR, Carrara $H A$, et al. Quality of life in women with breast cancer treated with or without chemotherapy. Cancer Nurs. 2017;40(2):108-16. DOI: https://doi.org/10.1097/NCC.0000000000000370

9. American Cancer Society. Breast Cancer Facts \& Figures 20152016. Atlanta: American Cancer Society; 2015

10. Ramírez K, Acevedo F, Herrera ME, Ibáñez C, Sánchez C. Actividad física y cáncer de mama: un tratamiento dirigido. Rev Médica Chile. 2017;145(1):75-84. DOI: https://doi.org/10.4067/S003498872017000100011

11. Soares Falcetta F, Araújo Vianna Träsel H, Almeida FK, Rangel Ribeiro Falcetta M, Falavigna M, Dornelles Rosa D. Effects of physical exercise after treatment of early breast cancer: systematic review and meta-analysis. Breast Cancer Res Treat. 2018;170(3):455-76.
12. Gomes NS, Soares MBO, Silva SR da. Autoestima e qualidade de vida de mulheres submetidas à cirurgia oncológica de mama. REME Rev Min Enferm. 2015;19(2):120-6. DOI: https://doi.org/10.5935/1415-2762.20150030

13. Hayes SC, Steele M, Spence R, Pyke C, Saunders C, Bashford J. Can Exercise influence survival following breast cancer? evidence from randomised, controlled trials. J Clin Oncol. 2017;35(Suppl 15):10067. DOI: https://doi.org/10.1158/1538-7445.SABCS17P6-12-01

14. Reis AD, Pereira PTVT, Diniz RR, Castro Filha JGL, Santos AM, Ramallo $B T$, et al. Effect of exercise on pain and functional capacity in breast cancer patients. Health Qual Life Outcomes. 2018;16(1):58. DOI: https://doi.org/10.1186/s12955-018-0882-2

15. Campbell KL, Van Patten CL, Neil SE, Kirkham AA, Gotay CC, Gelmon KA, et al. Feasibility of a lifestyle intervention on body weight and serum biomarkers in breast cancer survivors with overweight and obesity. J Acad Nutr Diet. 2012;112(4):559-67. DOI: https://doi.org/10.1016/j.jada.2011.10.022

16. Sedjo RL, Flatt SW, Byers T, Colditz GA, Demark-Wahnefried W, Ganz PA, et al. Impact of a behavioral weight loss intervention on comorbidities in overweight and obese breast cancer survivors. Support Care Cancer. 2016;24(8):3285-93. DOI: https://doi.org/10.1007/s00520-016-3141-2

17. Franklin DJ, Cheville AL. Medical Rehabilitation and the Palliative Care Patient. In: Oxford Textbook of Palliative Medicine. 5th ed. London: Oxford University Press; 2015:1-31. DOI: https://doi.org/10.1093/med/9780199656097.003.0415

18. Wurz A, St-Aubin A, Brunet J. Breast cancer survivors' barriers and motives for participating in a group-based physical activity program offered in the community. Support Care Cancer. 2015;23(8):2407-16. DOI: https://doi.org/10.1007/s00520-0142596-2

19. World Health Organization. Physical activity for health more active people for a healthier world: draft global action plan on physical activity 2018 - 2030. Geneva: WHO; 2018.

20. Kilgour RD, Jones DH, Keyserlingk JR. Effectiveness of a selfadministered, home-based exercise rehabilitation program for women following a modified radical mastectomy and axillary node dissection: a preliminary study. Breast Cancer Res Treat. 2008;109(2):285-95. DOI: https://doi.org/10.1007/s10549-0079649-x

21. Pinto BM, Rabin C, Dunsiger S. Home-based exercise among cancer survivors: adherence and its predictors. Psychooncology. 2009;18(4):369-76. DOI: https://doi.org/10.1002/pon.1465

22. Yang CY, Tsai JC, Huang YC, Lin CC. Effects of a home-based walking program on perceived symptom and mood status in postoperative breast cancer women receiving adjuvant chemotherapy. J Adv Nurs. 2011;67(1):158-68. DOI: https://doi.org/10.1111/j.1365-2648.2010.05492.x

23. Uhm KE, Yoo JS, Chung SH, Lee JD, Lee I, Kim JI, et al. Effects of exercise intervention in breast cancer patients: is mobile health (mHealth) with pedometer more effective than conventional program using brochure? Breast Cancer Res Treat. 2017;161(3):443-52. DOI: https://doi.org/10.1007/s10549-0164065-8

24. Matias GHL, Guerra ACCG, Souza Filho BAB, Lima JTO, Carmo CN, Mattos IE. Repetibilidade e reprodutibilidade de um manual de exercícios físicos domiciliares. Fisioter Pesqui. 2018;25(2):209-16. DOI: https://doi.org/10.1590/1809-2950/17010425022018

25. Instituto de Medicina Integral Professor Fernando Figueira. Informativo IMIP. Recife: IMIP; 2007.

26. Schulz KF, Altman DG, Moher D; CONSORT Group. CONSORT 2010 Statement: Updated guidelines for reporting parallel group randomised trials. J Clin Epidemiol. 2010;63(8):834-40. DOI: https://doi.org/10.1016/j.jclinepi.2010.02.005 
27. Schmitz KH, Courneya KS, Matthews C, Demark-Wahnefried W, Galvão DA, Pinto BM, et al. American College of Sports Medicine roundtable on exercise guidelines for cancer survivors. Med Sci Sports Exerc. 2010;42(7):1409-26. DOI: https://doi.org/10.1249/MSS.0b013e3181e0c112

28. Yesavage JA, Brink TL, Rose TL, Lum O, Huang V, Adey M, et al. Development and validation of a geriatric depression screening scale: a preliminary report. J Psychiatr Res. 1982-1983;17(1):3749. DOI: https://doi.org/10.1016/0022-3956(82)90033-4

29. Almeida OP, Almeida SA. Confiabilidade da Versão Brasileira da Escala de Depressão em Geriatria (GDS) versão reduzida. Arq Neuropsiquiatr. 1999;57(2B):421-6. DOI: https://doi.org/10.1590/S0004-282X1999000300013

30. Paradela EMP, Lourenço RA, Veras RP. Validation of geriatric depression scale in a general outpatient clinic. Rev Saude Publica. 2005;39(6):918-23. DOI: https://doi.org/10.1590/s003489102005000600008

31. World Health Organization. WHO Expert Committee on Physical Status: the use and interpretation of anthropometry. Geneva: WHO; 1995.

32. Alves RV, Mota J, Costa MC, Alves JGB. Aptidão física relacionada à saúde de idosos: influência da hidroginástica. Rev Bras Med Esporte. 2004;10(1):31-7. DOI: https://doi.org/10.1590/S151786922004000100003

33. Smethurst WS, Souza Filho BAB, Alves JGB, Silva Júnior JR. Ginástica para fazer em casa. Recife: IMIP; 2014.

34. Wolin KY, Schwartz AL, Matthews CE, Courneya KS, Schmitz KH. Implementing the exercise guidelines for cancer survivors. J Support Oncol. 2012;10(5):171-7. DOI: https://doi.org/10.1016/j.suponc.2012.02.001

35. Ghorayeb N, Stein R, Daher DJ, Silveira ADD, Ritt LEF, Santos DFPD, et al. The Brazilian Society of Cardiology and Brazilian Society of Exercise and Sports Medicine Updated Guidelines for Sports and Exercise Cardiology - 2019. Arq Bras Cardiol. 2019;112:326-368. DOI: https://doi.org/10.5935/abc.20190048

36. Jones CJ, Rikli RE. Measuring Functional. J Act Aging. 2002:24-30.

37. Dodds RM, Syddall HE, Cooper R, Benzeval M, Deary IJ, Dennison $E M$, et al. Grip strength across the life course: normative data from twelve British studies. PLoS One. 2014;9(12):e113637. DOI: https://doi.org/10.1371/journal.pone.0113637

38. Rikli RE, Jones CJ. Development and validation of a functional fitness test for community-residing older adults. J Aging Phys Act. 1999;7(2):129-61. DOI: https://doi.org/10.1123/japa.7.2.129

39. Rikli RE, Jones JC. Functional Fitness Normative Scores for Communit-Residing Older Adults, Ages 60-94. J Aging Phys Act. 1999;7(1):162-181. DOI: https://doi.org/10.1123/japa.7.2.162
40. Blair CK, Morey MC, Desmond RA, Cohen HJ, Sloane R, Snyder DC, et al. Light-intensity activity attenuates functional decline in older cancer survivors. Med Sci Sports Exerc. 2014;46(7):1375-83. DOI: https://doi.org/10.1249/MSS.0000000000000241

41. Vardar-Yagli N, Sener G, Saglam M, Calik-Kutukcu E, Arikan H, Inal-Ince $D$, et al. Associations among physical activity, comorbidity, functional capacity, peripheral muscle strength and depression in breast cancer survivors. Asian Pac J Cancer Prev. 2015;16(2):585-9. https://doi.org/10.7314/apjcp.2015.16.2.585

42. van Waart $H$, Stuiver MM, van Harten WH, Geleijn E, Kieffer JM, Buffart LM, et al. Effect of low-intensity physical activity and moderate- to high-intensity physical exercise during adjuvant chemotherapy on physical fitness, fatigue, and chemotherapy completion rates: results of the PACES randomized clinical trial. J Clin Oncol. 2015;33(17):1918-27. DOI: https://doi.org/10.1200/JCO.2014.59.1081

43. Murtezani A, Ibraimi Z, Bakalli A, Krasniqi S, Disha ED, Kurtishi I. The effect of aerobic exercise on quality of life among breast cancer survivors: a randomized controlled trial. J Cancer Res Ther. 2014;10(3):658-64. DOI: https://doi.org/10.4103/09731482.137985

44. Bertoli J. Efeitos do treinamento com o método Pilates de solo sobre variáveis neuromusculares e funcionais em mulheres idosas [Dissertação]. Florianópolis: Universidade Federal de Santa Catarina; 2016.

45. Loudon A, Barnett T, Piller N, Immink MA, Visentin D, Williams $A D$. The effects of Yoga on shoulder and spinal actions for women with breast cancer-related lymphoedema of the arm: a randomised controlled pilot study. BMC Complement Altern Med. 2016;16(1):343. DOI: https://doi.org/10.1186/s12906-0161330-7

46. Irwin ML. Guia do ACSM para exercício e sobrevivência ao câncer. São Paulo: Phorte; 2016.

47. Carminda MG, Rodolfo GC, Kristiane MBF, Pequeno LL, Pinheiro MHNP. Efeito do tempo de prática de exercício físico na aptidão física relacionada à saúde em mulheres idosas. Rev Bras Promoção Saúde. 2014;27(1):29-36. DOI: https://doi.org/10.5020/18061230.2014.p29

48. Booth FW, Roberts CK, Laye MJ. Lack of exercise is a major cause of chronic diseases. Compr Physiol. 2012;2(2):1143-211. DOI: https://doi.org/10.1002/cphy.c110025

49. Leach HJ, Danyluk JM, Nishimura KC, Culos-Reed SN. Benefits of 24 versus 12 weeks of exercise and wellness programming for women undergoing treatment for breast cancer. Support Care Cancer. 2016;24(11):4597-606. DOI: https://doi.org/10.1007/s00520-016-3302-3 